Herz 2012 · 37:7-11

DOI 10.1007/s00059-011-3573-x

Online publiziert: 21. Januar 2012

(c) Urban \& Vogel 2012

B. Maisch

Klinik für Innere Medizin, Schwerpunkt Kardiologie, Universitätsklinikum

Gießen und Marburg GmbH, Marburg

\title{
Kardiologische Rehabilitation - mehr als nur für den Schrebergarten?
}

pass-Chirurgie (ACVB) oder einer perkutanen transluminalen Intervention (PCI, Angioplastie oder Stentimplantation) sollten einer kardialen Rehabilitation zugeführt werden, die nach dem Aufenthalt in der Akutklinik stationär oder ambulant erfolgen kann (Klasse I, Evidenzgrad A). Diese Klasse-I-Empfehlung, allerdings mit dem schwächeren Evidenzgrad B, gilt für alle ambulant weiterbehandelnden $\mathrm{Pa}$ tienten auch innerhalb eines Jahres nach ACS, PCI, ACVB und chronischer (stabiler) Angina Pectoris (AP), sowie bei peripherer arterieller Verschlusskrankheit (PAVK). Kardiale Rehabilitation war historisch gesehen auch in Deutschland lange auf die Patienten mit KHK fokussiert. In diesen neuen Leitlinien wird eine dosierte körperliche Aktivität im Rahmen der Rehabilitation auch für Patienten mit stabiler Herzinsuffizienz als Klasse-IIaEmpfehlung mit dem Evidenzgrad B nahegelegt.

Diese durchaus ambitionierte Leitlinie fordert den Vergleich mit der deutschen Gegenwart heraus. Und dieser Aufgabe möchte sich Herz im ersten Heft des Jahres 2012 stellen. Für das Engagement, Themen und Autoren vorzuschlagen und selbst durch Beiträge mitzuwirken, danke ich besonders Herrn Dr. Rolf Dörr (Dresden) und Herrn Dr. Detlef Gysan (Köln).

\section{Sekundärprävention und Rehabilitation}

„Rehabilitation? - Nur für den Schrebergarten“, war der lapidare, oft etwas abschätzend gemeinte Kommentar vieler Kardiologen noch vor 30 Jahren. Denn die Wiedereingliederung in den Berufsalltag sollte zumindest aus Sicht des Rentenversicherungsträgers bei der Einführung der Anschlussheilbehandlung oder „Reha" im Vordergrund der ärztlichen Bemühungen stehen. Die kardiale Rehabilitation stand damals erst am Anfang ihrer Validierung. Das wurde im Laufe der Jahre anders. Kardiale Rehabilitation nach einem kardiovaskulären Ereignis wurde zu einem festen Bestandteil kardiologischer Therapiekonzepte nach Herzinfarkt und Bypass-Operation. Es dauerte allerdings 2 Jahrzehnte bis die Europäischen Gesellschaft für Kardiologie (ESC) in ihrer Deklaration von Barcelona die Absicht festschrieb, dass „,jeder Patient nach einem akuten kardiovaskulären Ereignis zumindest einmal in seinem Leben die Chance haben sollte, ein kardiovaskuläres Präventions- und Rehabilitationsprogramm zu besuchen“.

Die WHO im Jahr 1993 und kurze Zeit später auch die Deutsche Gesellschaft für Kardiologie(DGK), die Deutsche Gesellschaft für Kardiale Prävention und Rehabilitation (DGPR) und die Deutsche Gesellschaft für Herzchirurgie machten sich dieses Anliegen in Form einer eigenen Leitlinie zu eigen: Die kardiologische Rehabilitation wurde zu einem“integralen Bestandteil einer am langfristigen Erfolg orientierten, umfassenden Versorgung von Herzpatienten“ erklärt $[2,3]$.

Stiefkind bleibt allerdings auch bei uns bis heute die Primärprävention, die in erster Linie auf die Mitwirkung der noch Gesunden setzt. Denn auch heute noch stellen Herz-Kreislauf-Krankheiten in Deutschland die häufigste Todesursa- handlung eines akuten Koronarsyndrom (ACS), direkt nach aortokoronarer By- 


\section{Editorial}

Tab. 1 Leitlinien zur Sekundärprävention und Risikoreduktion (nach [1])

\begin{tabular}{|c|c|c|c|}
\hline Intervention & Ziel & $\begin{array}{l}\text { Empfehlung } \\
\text { Klasse }\end{array}$ & $\begin{array}{l}\text { Evidenz- } \\
\text { grad }\end{array}$ \\
\hline Rauchen & Verzicht, auch auf Passivrauchen & I & B \\
\hline Blutdruck & $<140 / 90 \mathrm{~mm} \mathrm{Hg}$ & I & $\begin{array}{l}B \text { für Diät } \\
\text { A für BB, ACE }\end{array}$ \\
\hline \multirow[t]{7}{*}{ Lipidstatus } & $\begin{array}{l}\text { Lipidsenkung durch Lebensstilmodifikation, Diät mit gesättigte FS <7\%, } \\
\text { Cholesterin }<200 \mathrm{mg} / \mathrm{Tag}\end{array}$ & I & B \\
\hline & Statintherapie & I & A \\
\hline & $\mathrm{LDL}-\mathrm{C}<100 \mathrm{mg} / \mathrm{dl}$ & I & $\mathrm{C}$ \\
\hline & Triglyzeride $<200 \mathrm{mg} / \mathrm{dl}$ & I & B \\
\hline & Bei Cholesterin >200 mg/dl und Statinunverträglichkeit: Niacin & Ila & B \\
\hline & Ezetimibe bei CSE-Unverträglichkeit & $\mathrm{llb}$ & $\mathrm{C}$ \\
\hline & Omega-3-Fettsäuren (1 g/Tag) & $\mathrm{Ilb}$ & B \\
\hline Körperliche Aktivität & $\begin{array}{l}\text { Mindestens } 30 \text { min an mindestens } 5 \text { Tagen/Woche mit Information des } \\
\text { behandelnden Arztes }\end{array}$ & I & B \\
\hline Gewichtskontrolle/-reduktion auf & $\begin{array}{l}\text { BMl: } 18,5-24,0 \mathrm{~kg} / \mathrm{m}^{2} \\
\text { Hüftumfang: Frauen }<89 \mathrm{~cm} \text {, Männer }<102 \mathrm{~cm}\end{array}$ & I & B \\
\hline \multirow[t]{3}{*}{ Typ-II-Diabetes-Management } & $\begin{array}{l}\text { Lebensstiländerung und Koordination des Endokrinologen mit dem } \\
\text { Allgemeinmediziner/Internisten }\end{array}$ & I & B \\
\hline & $\begin{array}{l}\text { Metformin als,"First-line-Therapie“ } \\
\text { oder individualisiert }\end{array}$ & $\begin{array}{l}\text { Ila } \\
\text { Ila }\end{array}$ & $\begin{array}{l}\text { A } \\
C\end{array}$ \\
\hline & Ziel-HbA1c $<7 \%$ & $\mathrm{llb}$ & $\mathrm{C}$ \\
\hline $\begin{array}{l}\text { Thrombozytenaggregations- } \\
\text { hemmung }\end{array}$ & $\begin{array}{l}\text { Aspirin } 75-162 \mathrm{mg} \\
\text { bei Unverträglichkeit Clopidogrel } 75 \mathrm{mg}\end{array}$ & $\begin{array}{l}1 \\
1\end{array}$ & $\begin{array}{l}\text { A } \\
B\end{array}$ \\
\hline $\begin{array}{l}\text { Duale Thrombozytenaggrega- } \\
\text { tionshemmung }\end{array}$ & $\begin{array}{l}\text { Bei ACS, bei BMS oder DES Aspirin und Thiopyridin oder Prasugrel } 10 \mathrm{mg} \text { oder } \\
\text { Ticagrelor } 90 \mathrm{mg} \text { über } 12 \text { Monate }\end{array}$ & I & B \\
\hline \multirow[t]{2}{*}{$\begin{array}{l}\text { RAAS-Blockade } \\
\text { mit ACEI }\end{array}$} & $\begin{array}{l}\text { Alle Patienten mit EF } \leq 40 \% \text { oder Hochdruck, Diabetes, chron. Nierenerkrankung, } \\
\text { falls keine Kontraindikation besteht }\end{array}$ & I & A \\
\hline & Alle übrigen Patienten & Ila & B \\
\hline \multirow[t]{2}{*}{ mit ARBs } & Bei ACEI-Intoleranz bei EF $\leq 40 \%$ & I & A \\
\hline & Alle übrigen Patienten bei ACEI-Intoleranz & Ila & B \\
\hline mit Aldosteronblockade & $\begin{array}{l}\text { Alle Patienten nach Infarkt mit } E F \leq 40 \text {, die bereits ACEl und BB haben, oder mit } \\
\text { Diabetes oder Herzinsuffizienz }\end{array}$ & I & A \\
\hline \multirow[t]{4}{*}{ Betablocker } & Alle Patienten bei EF $\leq 40 \%$ postinfarkt oder mit Herzinsuffizienz ohne KI & I & A \\
\hline & Nach Infarkt oder bei ACS bei normaler EF für 3 Jahre & I & B \\
\hline & Nach Infarkt oder bei ACS bei normaler EF für mehr als 3 Jahre & Ila & B \\
\hline & Bei Pat. mit EF $\leq 40 \%$ ohne ACS oder MI & Ila & $\mathrm{C}$ \\
\hline Jährliche Influenza-Schutzimpfung & Alle Herzpatienten & I & B \\
\hline Depression & Alle Pat. nach ACVB oder MI & Ila & B \\
\hline \multirow{4}{*}{$\begin{array}{l}\text { Kardiale Reha ambulant, heimat- } \\
\text { nah oder im Zentrum }\end{array}$} & Alle Pat. direkt nach ACS, direkt nach ACVB oder PCI & I & A \\
\hline & Alle ambulanten Pat. innerhalb eines Jahres nach ACS, ACVB oder PCl oder bei PAVK & I & B \\
\hline & Alle ambulanten Pat. mit chronischer AP & I & B \\
\hline & Pat. mit stabiler Herzinsuffizienz zur kontrollierten körperlichen Aktivität & Ila & B \\
\hline
\end{tabular}

che dar. Bereits vor Erreichen des Rentenalters mit dem 65. Lebensjahr gingen, so Korsukewitz, Falk und Lindow in ihrem Beitrag in dieser Ausgabe von Herz zur Bilanz der deutschen Rentenversiche- rung, im Jahre 201028.400 sog. vorzeitige Todesfälle zu Lasten von Herz-Kreislauf-Erkrankungen. Die durch die KHK verlorenen Lebensjahre, d. h. die Differenz zwischen dem 65. Lebensjahr und dem vorzeitigen Versterben infolge kardiovaskulärer Erkrankungen, summierten sich im Jahre 2010 für Männer auf 95.000 und für Frauen auf 21.000 Jahre. Die krankheitsbedingten Ausfälle der Er- 
werbstätigkeit der Bevölkerung im Alter von 15 bis 64 Jahren in Form von „verlorenen“ Erwerbstätigkeitsjahren infolge von Arbeitsunfähigkeit, Invalidität und Tod durch Herz-Kreislauf-Erkrankungen waren erwartungsgemäß noch größer und betrugen in Deutschland 382.000 Erwerbstätigkeitsjahre. Diese Zahlen stecken den Rahmen der Aufgaben ab, die sich dem Rentenversicherungsträger stellen, wenn er die Wiederherstellung der Arbeitsfähigkeit durch die kardiale Rehabilitation als primäre Aufgabe definieren würde. Die Anforderungen zur kardiovaskulären Sekundärprävention jenseits der Berentung oder Pensionierung sind noch wesentlich größer. Sie umfassen auch die in den letzten 30 Jahren um etwa 7 Jahre älter gewordenen und polymorbiden Patienten, für die die Rehabilitation "aus dem Schrebergarten für den Schrebergarten“ der Ausgangspunkt war. Hier gilt es, altersspezifische Reha-Konzepte $\mathrm{zu}$ entwickeln und das Potenzial der kardiologischen Rehabilitation auch außerhalb des klassischen AHB-Verfahrens zu erkennen und zu nutzen.

Die kardiologische Rehabilitation der Rentenversicherung, so Korsukewitz und Kollegen, kann deshalb durchaus auf eine von Paradigmenwechseln geprägte Vergangenheit zurückblicken. Sie stellt aber gerade auch deshalb ein „Erfolgsmodell mit Perspektive" dar.

H.W. Hahmann benennt in seinem Beitrag „Kardiologische Rehabilitation - gestern, heute und morgen " die geänderten Ausgangsbedingungen: Der demographische Wandel von den Berufstätigen zu den Rentnern ist Realität, denn der größte Teil kardiologischer Reha-Patienten ist bereits oder wird nach der „Reha“ berentet. Kardiale Ereignisse, die zur Rehabilitation führen, finden heute bevorzugt im Rentenalter statt. Aber die Rehabilitanden sind eben nicht nur älter, sondern auch kränker. Dennoch werden diese Patienten vermehrt aus der Akutklinik einer frühen Rehabilitation zugeführt - dies als Folge des Kostendrucks in den erstbehandelnden Krankenhäusern. So wurde mit der kürzeren stationären Verweildauer die „blutige Verlegung“" zum Schlagwort.

Während zu Beginn der Entwicklung von Reha-Konzepten der Nutzen einer kardialen Rehabilitation nur postu- liert werden konnte, wurden ihre Erfolge mit den Jahren zunehmend besser belegt: Ein Beispiel von Belang ist die randomisierte, placebokontrollierte doppelblinde OMEGA-Studie mit 3.851 Patienten nach PCI bei akutem Koronarsyndrom (STEMI oder NSTEMI), von denen $70,6 \%$ an einer kardiologischen Anschlussrehabilitation teilnahmen. Die Ereignisraten zeigten einen signifikanten Vorteil der Rehabilitation hinsichtlich Gesamtmortalität, überlebtem Myokardinfarkt und dem Auftreten einer Herzinsuffizienz [5, 6].

In ihrem Beitrag zur ambulanten Rehabilitation belegen Fischer, Charrier und Dörr, dass die wohnortnahe ambulante Rehabilitation sich in den letzten Jahren gleichberechtigt neben der stationären Rehabilitation etabliert hat. Die ambulante „Reha“ ermöglicht eine enge Einbindung des sozialen und familiären Umfelds des Patienten und fördert so eine Reintegration in den Alltag. Allerdings wird das Potenzial der ambulanten Rehabilitation von den Kostenträgern und den $\mathrm{Pa}$ tienten in Deutschland noch nicht ausreichend genutzt.

Wie in Deutschland ist auch in der Schweiz für die kardiologische Rehabilitation ein deutlicher Trend zur ambulanten Rehabilitationsform festzustellen. In seinem Beitrag weist Hugo Saner darauf hin, dass Kostendruck und geänderter Bedarf in der Schweiz zur Schließung stationärer und zur Bevorzugung ambulanter RehaEinrichtungen geführt haben.

In diesem Heft greifen wir wegen des Themenreichtums erstmals auch zu Spotlight-Beiträgen, um in prägnanter Form schlaglichtartig auf aktuelle Entwicklungen und Trends hinzuweisen.

Penzel, Fietze, Schöbel und Baumann zeigen in ihrem Beitrag, dass sich schlafbezogene Atmungsstörungen häufig auch bei Patienten in der kardiologischen Rehabilitation finden und einen bedeutenden Risikofaktor für kardiovaskuläre Erkrankungen darstellen.

G. Bönners Beitrag fokussiert auf eine Patientengruppe mit besonders hohem Risiko: die Diabetiker. Sie waren in schöner Regelmäßigkeit Thema von Schwerpunktheften von Herz [7, 8, 9]. Ihre Prävention benötigt zusätzliche Aufmerksamkeit [10]. Sie benötigen spezielle Rehabilitationsprogramme, in deren Vor- dergrund die Lebensstilanpassung an die Krankheit steht. Diabetes mellitus ist nicht nur der Schrittmacher für eine koronare und periphere Makro- und Mikroangiopathie, sondern auch für die diabetische Kardiomyopathie $[11,12,13]$. Eine adäquate Therapie der Hypertrophie ist eine permanente Herausforderung, gerade wenn der Risikofaktor Hochdruck dazukommt [14].

J. Schaefer versucht in seinem Kurzbeitrag die Frage zu beantworten, ob hohes HDL per se gesund ist oder ob Gesunde "nur" ein hohes HDL haben. Diese Frage ist bei den negativen Ergebnissen der ILLUMINATE-Studie [15] durch die Behandlung mit CETP-Inhibitoren, die HDL erhöhen, ohne die Prognose zu verbessern, gegenwärtig von besonderer Aktualität.

Mit CorBene stellen Gysan, Albus, Riedel, Hossmann, Vogt, Latz und Griebenow ein neues Versorgungsmodell vor, um Lebensqualität und Prognose von Patienten mit Herzinsuffizienz zu verbessern und die Hospitalisierungsrate zu vermindern. Ihr Merkmal ist die enge Zusammenarbeit zwischen Hausarzt, niedergelassenem Kardiologen, Krankenhauskardiologen und Rehabilitationseinrichtung.

Albus, Bjarnson-Wehrens, Gysan, Herold, Schneider, Eulenburg und Predel zeigen in der randomisierten, kontrollierten Präford-Studie an 447 Männern und Frauen mit einem ESC-Risiko-Score von über 5\% überraschend, dass eine multimodale Intervention auf Depressivität im Vergleich zu einer Routineversorgung keine Verbesserung der Depressivität zur Folge hat. Dabei bleibt unbestritten, dass die Depression ein wichtiges Begleitsymptom des kardiovaskulären Krankheitsspektrums von der KHK bis zur Herzinsuffizienz darstellt [16].

B. Schwabs Beitrag beantwortet die Frage, ob telemedizinische Studien zu häuslichem Ergometertraining mit EKGMonitoring und Internet-basierte Schulungen zur Lebensstilmodifikation ausreichen, zunächst abschlägig. Bei ausgewählten Patienten leistet Telemonitoring zwar einen wertvollen Beitrag, kann aber die multimodale Rehabilitation nicht ersetzen.

Die unterschiedlichen Ergebnisse zweier großer telemetrischer Studien, der 
positiven München- [17] und der neutral verlaufenden TIM-HF-Studie bei chronischer Herzinsuffizienz [18] veranlassen M. Middecke zu der Empfehlung, dass nach stationärem Aufenthalt wegen dekompensierter Herzinsuffizienz eine weiterführende telemedizinische Betreuung besonders dann sinnvoll sein kann, wenn die maximale medikamentöse Therapie noch nicht ausgeschöpft wurde.

Werner, Fürster und Laufs weisen in ihrem Beitrag über körperliche Aktivität und myokardiales Remodelling darauf hin, dass moderate körperliche Aktivität ein wichtiger Bestandteil der Primär- und Sekundärprävention kardiovaskulärer Erkrankungen sein sollte. Bei Patienten mit manifesten Herz-Kreislauf-Erkrankungen ist eine Anpassung der Trainingsempfehlung an die zugrunde liegende Myokarderkrankung und an individuelle Risikofaktoren indiziert.

Ein nicht ausreichend wahrgenommenes Thema mit erheblicher sozialer Brisanz ist die „Verkehrstauglichkeit als Autofahrer" eines Reha-Patienten nach Herzinfarkt, dem sich Th. und I.G. Wendt aus juristischer und medizinischer Sicht zuwenden. Dem niedergelassenen Arzt ist nicht bewusst - und noch weniger dem Klinikarzt -, dass selbst nach einem unkompliziert verlaufenen Infarkt der Gesetzgeber eine mindestens 3-monatige Abstinenz vom Führen eines Fahrzeugs fordert.

\section{Primärprävention}

Die Primärprävention kardiovaskulärer Erkrankungen ist die logische Voraussetzung zur Vermeidung kardiovaskulärer Ereignisse, die eine Sekundärprävention erforderlich machen. Hier sind jedoch die Erfolge einer Intervention wesentlich weniger gut belegt. In seinem Beitrag „Primärprävention der koronaren Herzerkrankung: Was nützen die Risiko-Scores?" geht H. Gohlke auf den PROCAM-Algorithmus und den ESC-SCORE ein. Wenn der Grenzwert zu einem hohen Risiko überschritten wird, ist eine medikamentöse Therapie kosteneffektiv, und die „number needed to treat" (NNT), normiert auf 1 Jahr, liegt unter 200. Auch bei einzelnen, stark ausgeprägten Risikofaktoren wird eine NNT von unter 200 erreicht. Le- bensstilfaktoren werden im CARRISMASystem zusätzlich zur Risikostratifizierung genutzt und ergeben Ansatzpunkte für die Therapie. Da sich die mittlere Lebenserwartung und die Präventionsmöglichkeiten verbessert haben, sollte eine auf Scores basierende Risikostratifikation der Patienten bis zum 70. Lebensjahr erfolgen, damit eine evidenzbasierte und kosteneffektive Prävention auch im höheren Alter erfolgen kann.

Eine rechtzeitige und sinnvolle Primärprävention würde die Sekundärprävention in der Rehabilitation überflüssig machen oder zumindest weiter hinauszögern. Primärprävention wäre dann ein Weg zur Verhinderung der „Rehabilitation für den Schrebergarten “ - und dies im besten Interesse der Menschen.

Bernhard Maisch

\section{Korrespondenzadresse}

\section{Prof. Dr. B. Maisch}

Klinik für Innere Medizin,

Schwerpunkt Kardiologie,

Universitätsklinikum Gießen und

Marburg GmbH

Baldingerstr. 1, 35033 Marburg

maisch@staff.uni-marburg.de

\section{Literatur}

1. Smith SC, Benjamin EJ, Bonow RO et al (2011) AHA/ACCF secondary prevention and risk reduction therapy for patients with coronary and other atherosclerotic vascular disease: 2011 update: a guideline from the American Heart Asscoaition and American College of Cardiology Foundation. Circulation 124(22):2458-2473

2. World Health Organisation Expert Committee (1993) Rehabilitation after cardiovascular diseases, with special emphysis on developing countries. WHO Technical Report Series 831

3. Dietz R, Rauch B (2003) Leitlinie zur Diagnose und Behandlung der chronischen koronaren Herzerkrankung der Deutschen Gesellschaft für Kardiologie - Herz- und Kreislaufforschung (DGK). In Kooperation mit der Deutschen Gesellschaft für Prävention und Rehabilitation von Herz-Kreislauferkrankungen (DGPR) und der Deutschen Gesellschaft für Thorax-, Herz- und Gefäßchirurgie (DGTHG). Z Kardiol 92:501-521

4. Bjarnason-Wehrens B, Held K, Hoberg E et al (2007) Deutsche Leitlinie zur Rehabilitation von Patienten mit Herz-Kreislauferkrankungen (DLLKardReha). Clin Res Cardiol Suppl 2: III/1-III/54
5. Rauch B, Schiele R, Schneider S et al, for the OMEGA Study Group (2011) OMEGA, a randomized, placebo-controlled trial to test the effect of highly purified omega-3 fatty acids on top of modern guideline-adjusted therapy after myocardial infarction. Circulation 122:2152-2159

6. Schwaab B, Riemer T, Schneider S et al (2011) Cardiac rehabilitation after acute myocardial infarction - effect on mortality, morbidity, medication and lifestyle changes. Eur Heart J 32 (Abstr Suppl), 389

7. Ryden I, Tschöpe D, Dörr R (2008) Diabetes and the heart - revisited (Editorial). Herz 33:169

8. Dörr R, Müller-Wieland D, Tschöpe D (2010) Diabetes and the heart - a never-ending story (Editorial). Herz 35:129

9. Schneider CA, Pfister R, Erdmann E (2010) Diabetes und Herzinsuffizienz. Herz 35:140-147

10. Anselmino M, Gohlke H, Mellbin L, Ryden L (2008) Cardiovascular prevention in patients with diabetes and prediabetes. Herz 33:170-177

11. Saunders J, Mathewkutty S, Drazner MH, McGuire DK (2008) Cardiomyopathy in type 2 diabetes. Update on pathophysiologcal mechanisms. Herz 33:184-190

12. Stratmann B, Gawlowski T, Tschöpe D (2010) Diabetic cardiomyopathy - to take a long story serious. Herz 35:161-169

13. Maisch B, Alter P, Pankuweit S (2011) Diabetic cardiomypathy - fact or fiction? Herz 36:102-115

14. Holzgreve $H$ (2008) Die Behandlung des Typ-2-Diabetikers mit Hypertonie: zu spät und zu wenig. Herz 33:191-195

15. Barter PJ, Caulfield M, Eriksson M et al; ILLUMINATE Investigators (2007) Effects of torcetrapib in patients at high risk for coronary events. N Engl J Med 357:2109-2122

16. Herrmann-Lingen C (2011) Psychosomatik der Herzinsuffizienz. Alles nur Depression? Herz 36:135-141

17. Kielblock B, Frye Ch, Kottmair S et al (2007) Einfluss einer telemedizinisch unterstützten Betreuung auf Gesamtbehandlungskosten und Mortalität bei chronischer Herzinsuffizienz. Dtsch Med Wochenschr 132(9):417-422

18. Koehler F, Winkler S, Schieber M et al (2011) Impact of remote telemedical management on mortality and hospitalizations in ambulatory patients with chronic heart failure. Circulation 123:18731880 
Hier steht eine Anzeige.

黛 Springer 